\title{
Preliminary Computational Assessment of Disk Rotating Detonation Engine Configurations
}

\author{
Daniel E. Paxson \\ NASA Glenn Research Center \\ Cleveland, Ohio \\ JANNAF Joint Subcommittee Meeting \\ $37^{\text {th }}$ Airbreathing Propulsion Subcommittee \\ Dayton, Ohio \\ June 3-7, 2019
}




\section{Outline}

- Background

- Modeling Approach

- Simple Tests

- Results

- Concluding Remarks 


\section{Background}

The Pressure Gain Combustion Community is Investigating Rotating Detonation Engine (RDE) Configurations Where Flow is Radial

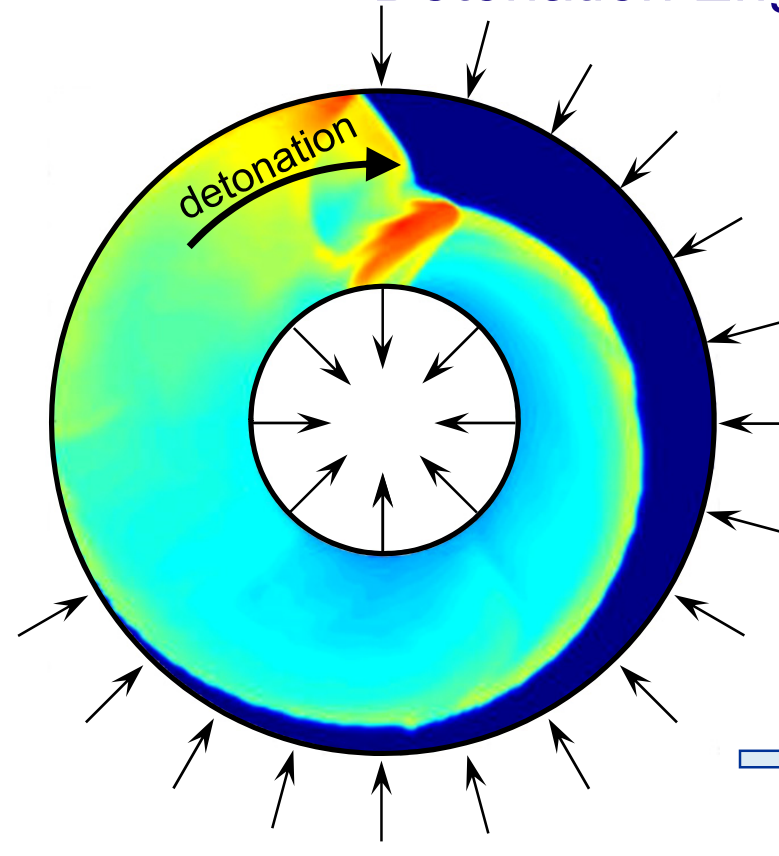

Top view inward flow

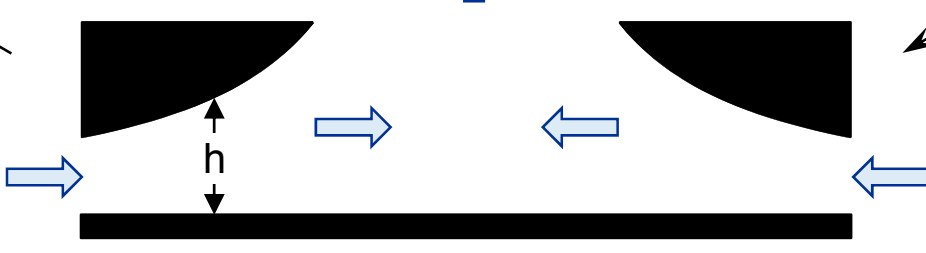

Side view constant area

- Inward and outward flow scenarios are of interest

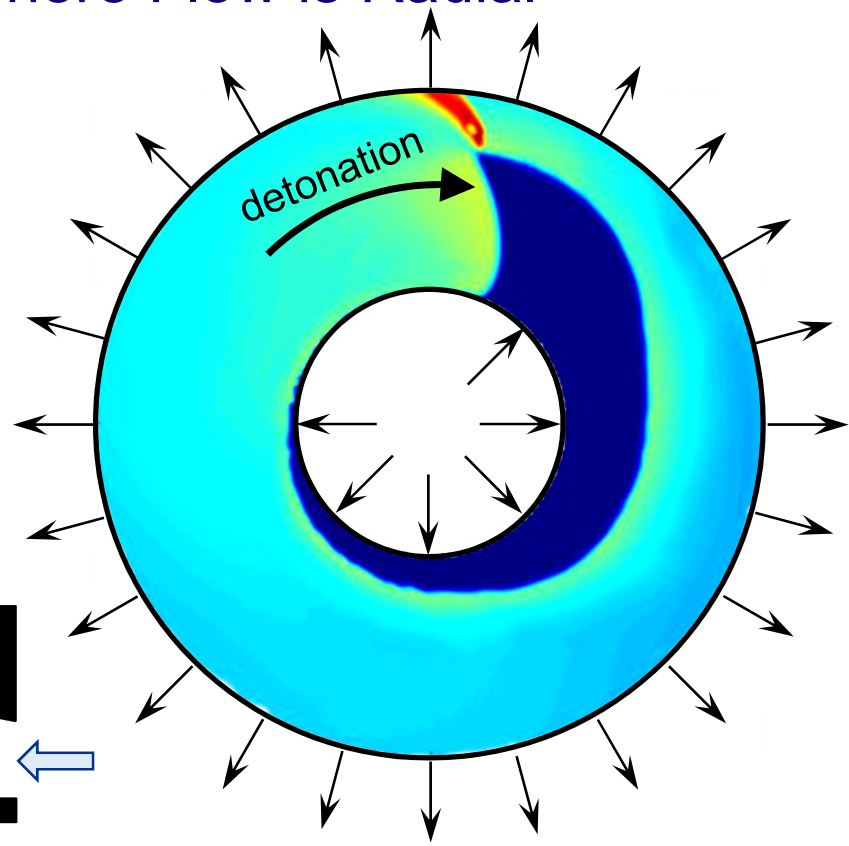

Top view outward flow

- Compact

- Intuitively well-matched to radial turbomachinery

- May enhance detonative cycle performance

- Centrifugal forces may be of benefit

\section{Fast, Flexible Simulation Capability Is Needed}




\section{Modeling Approach}

\section{Use the Exact Same Q2D Methodology Currently Employed for Annular RDE's}

\section{Benefits:} (Distr. C Released LEW-19488-1)

- Regularly spaced Cartesian grid keeps code simple and fast (runs in minutes on a laptop)

- Good for basic parametric studies

- No core code development required Challenges:

- Necessitates dropping the detonation frame of reference

- Results in shocks at high skew angles to grid

- Boundary surface areas are $>\pi \mathrm{d}$

- Boundary conditions are required in both $x$ and $y$ directions

- Boundary cells (aka, ghost cells) are not regularly spaced

- Inflow boundaries require that flow is radial (much algebra in a Cartesian system)

- No analytical 'test cases' to validate

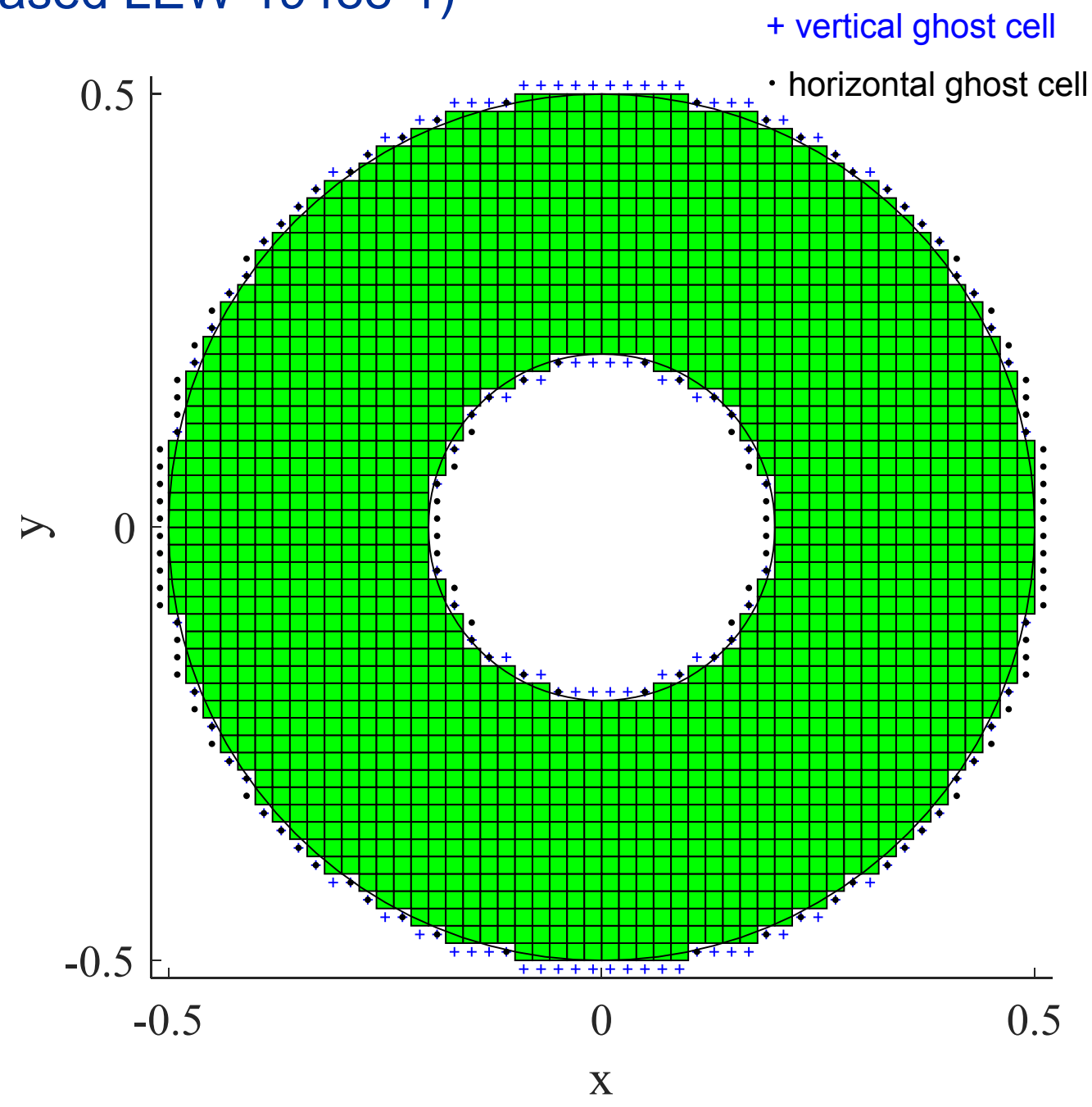

Challenges Are Mostly Bookkeeping, Approach is Sound 


\section{Simple Non-Reactive 'Shock Tube' Test}

Setup

-200 X 200 grid-no height variation (parallel plates)

-Walls at inner and outer diameter; $D_{j} / D_{0}=0.5$

- Intial state (non-dimensional): $p, \rho, u, v, z=1,1,0,0,0$ everywhere except in a rectangle at bottom of disk where $p, \rho=10,10$

- Simulation time is 0.8 units $\left(\mathrm{t} \times \mathrm{a}^{*} / \mathrm{D}_{\mathrm{o}}\right)$

Results

-Waves move at the correct speed

- Shocks have the correct curvature

- Symmetry is proper

- 'Stair Step' walls are rough but acceptable
CFD Video Showing Contours of Temperature

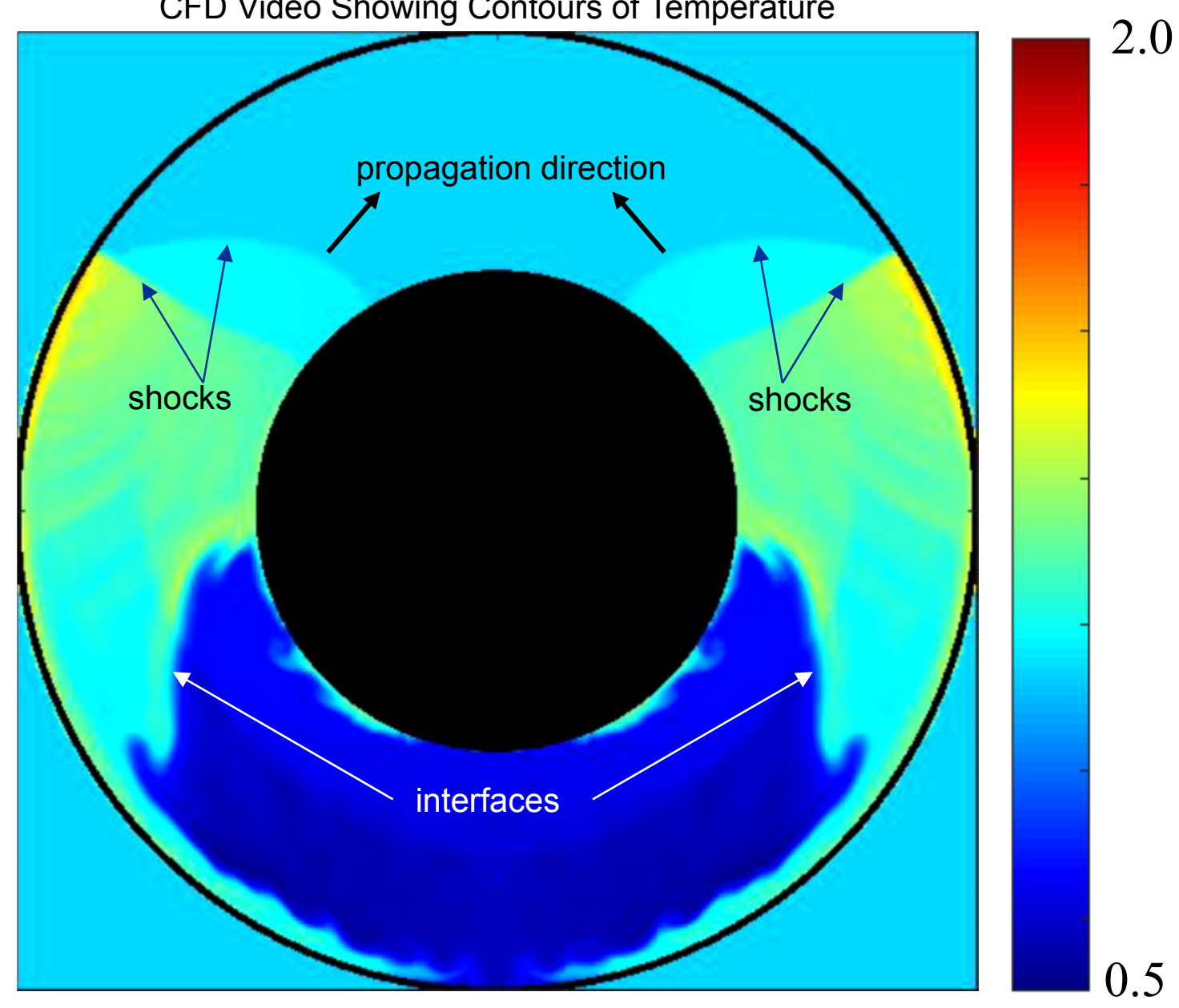

Wall B.C. and Cartesian Grid Appear to Capture Basic Waves 


\section{Simple $\mathrm{H}_{2}$ /Air One-Shot Detonation Test}

Setup

- $200 \times 200$ grid - no height variation (parallel plates)

- Walls at inner and outer diameter

- Initial state (non-dimensional): $p, \rho, u, v, z=1,1,0,0,1$ everywhere except in a square at bottom of disk where $p, \rho, z=17.0,1.745$, 0.0

- Simulation time is 0.25 units Results

- Detonation speed is nominally correct

- Curvature of detonation and uniform angular velocity indicate circumferential velocity is different everywhere

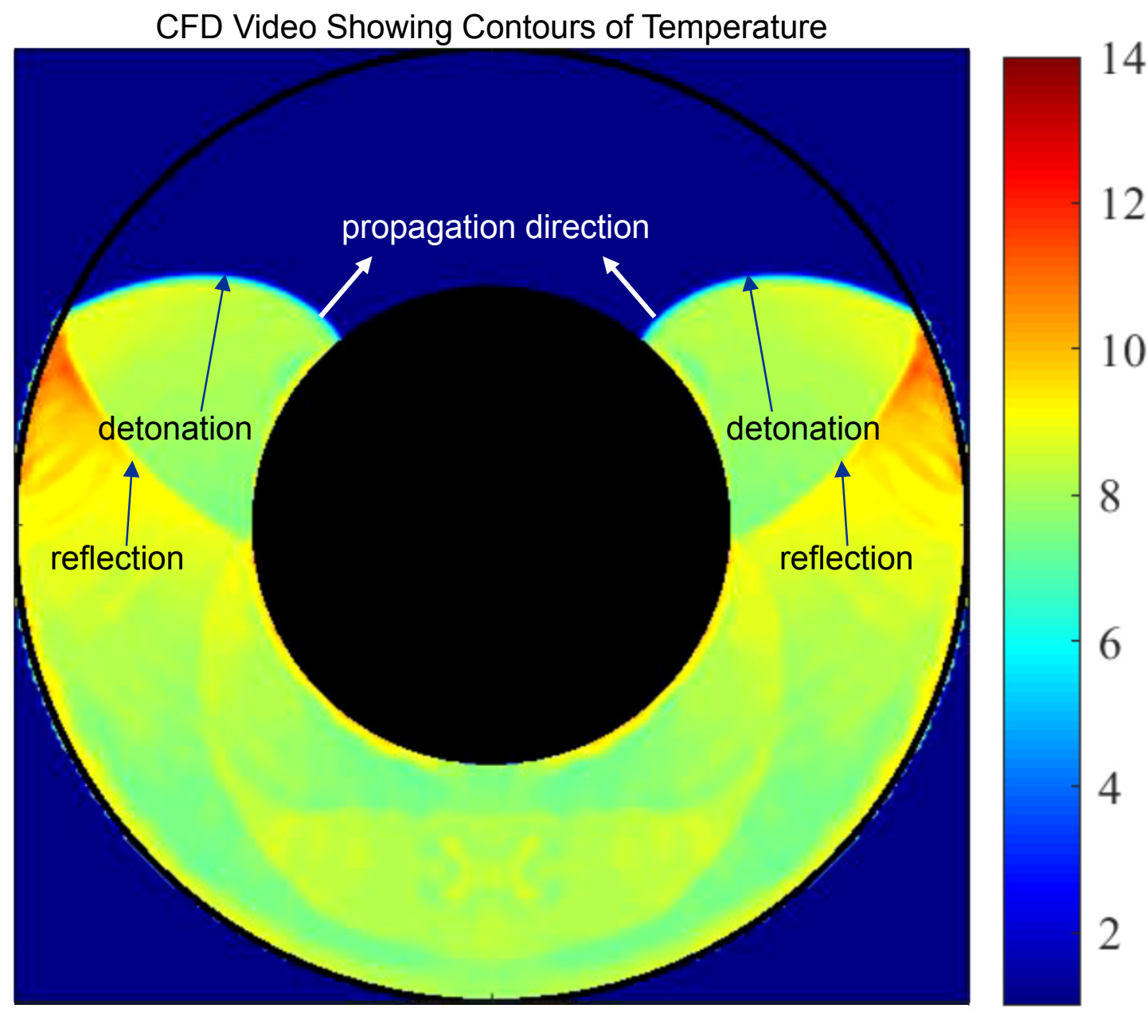

- Laboratory frame of reference works

Reaction Model Successful for This Configuration 


\section{Simple Shock Wave Inflow and Outflow Test}

Setup

- $200 \times 200$ grid - no height variation (parallel plates)

- Radial inflow at outer diameter; constant pressure at inner diameter

- $p, \rho, u, v, z=1,1,0,0,0$ everywhere

- Inner diameter $p=1.0$; Outer manifold $p, T=2.0,1.03846$

- Simulation time is 1.0 units Results

- Wave speeds nominally correct

- Inflow and outflow mass flow rates match after 1.2 units

- Inflow is radial (on a Cartesian grid!)

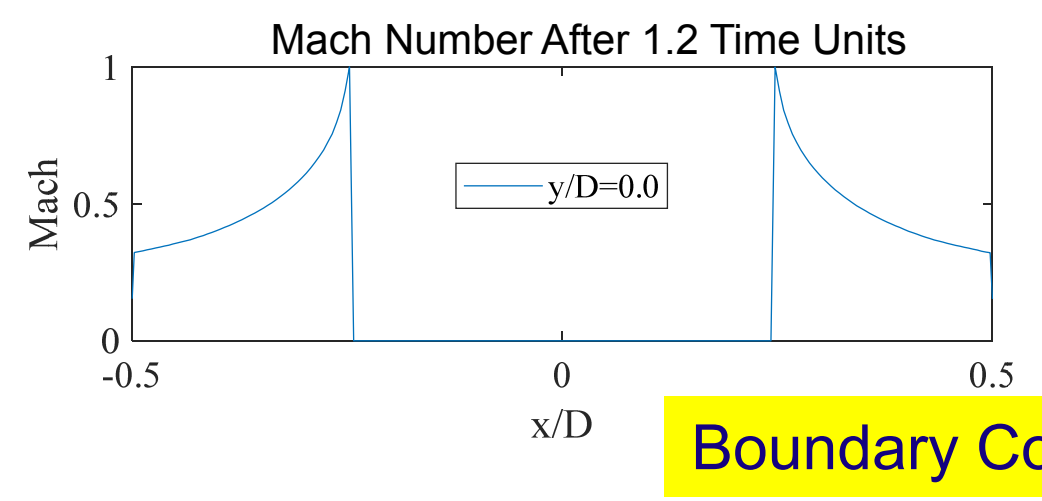

Inflow Must Be Radial

CFD Video Showing Contours of Pressure

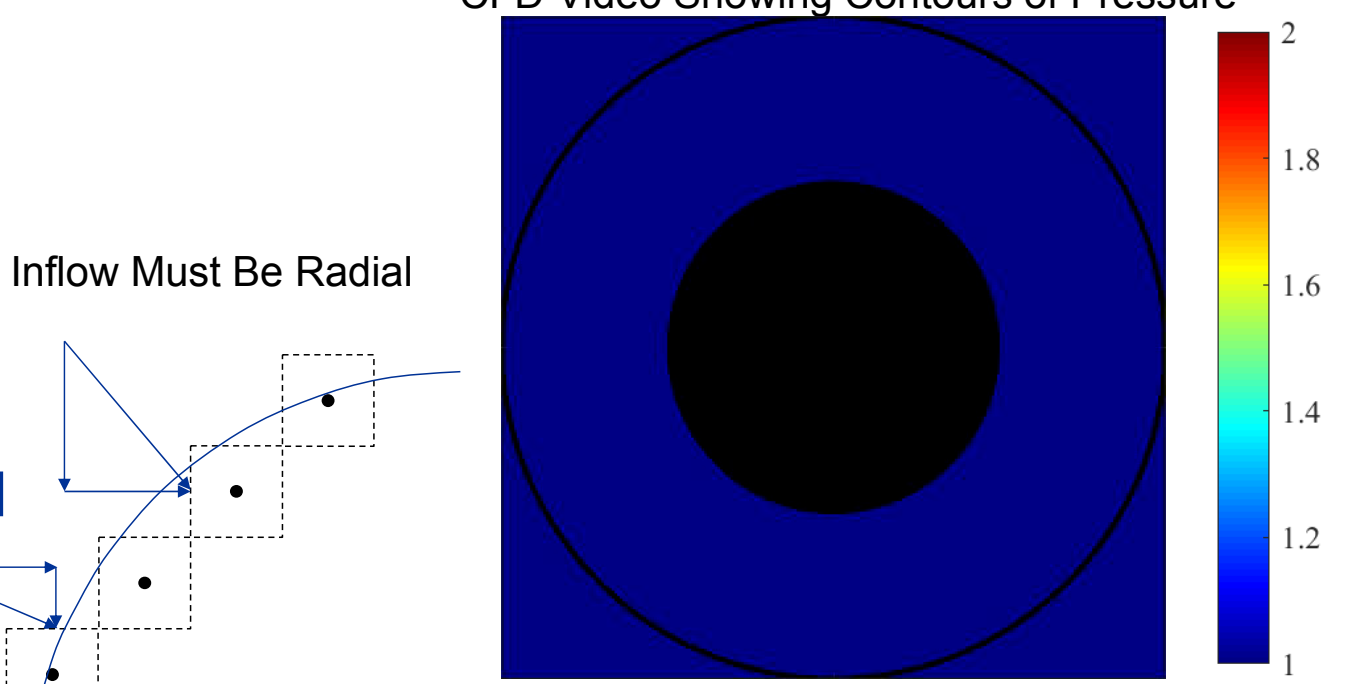

Contours of Pressure and Streamlines After 1.2 Time Units

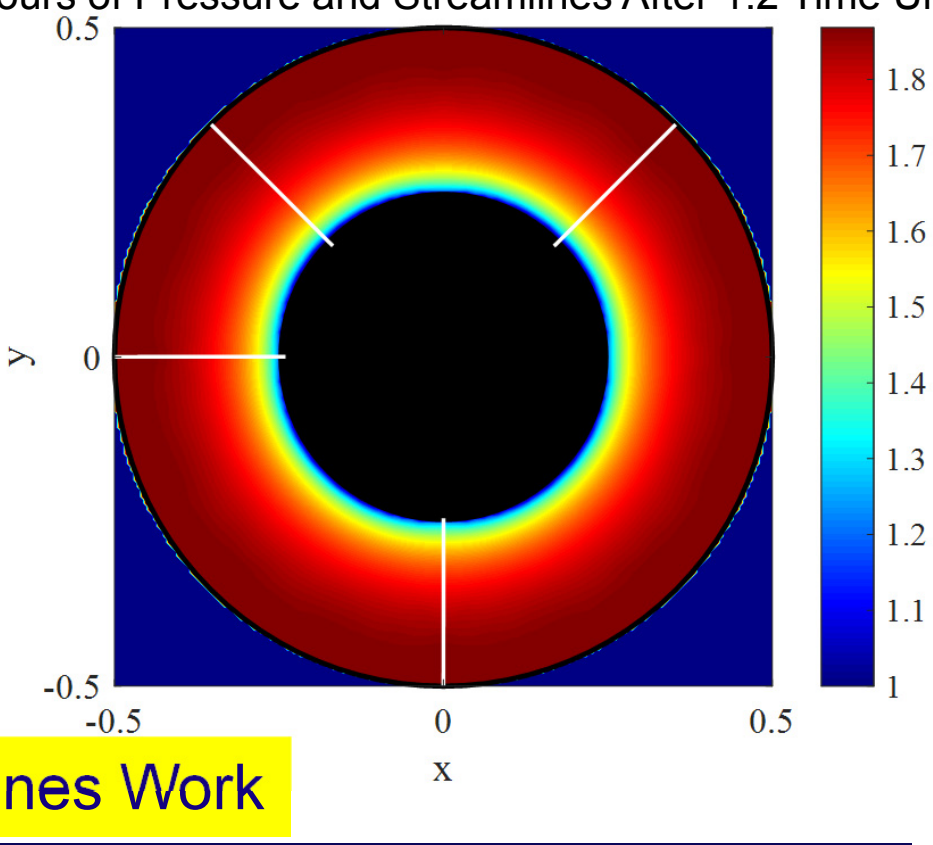


RDE Results: $\mathrm{H}_{2}$ /Air; Radially Inward (NOTE: All Results Are $200 \times 200$ Grid)

Setup

- Grid-height variation keeps area constant

- $D_{\mathrm{i}} / \mathrm{D}_{\mathrm{o}}=0.5 ; \mathrm{A}_{\mathrm{in}} / \mathrm{A}_{\mathrm{ch}}=1.0$; Inlet check valve

- Boundary Conditions:

- Outer manifold p, T = 4.0, 1.03846

- Inner diameter $p=1.0$

- Video shows 0.52 time units; started after approximately 3 wave revolutions

Results

- Detonation speed $10 \%$ above CJ based on OD

- Detonation is unstable and ultimately fails

- Annular RDE is stable with these lossless boundary and conditions

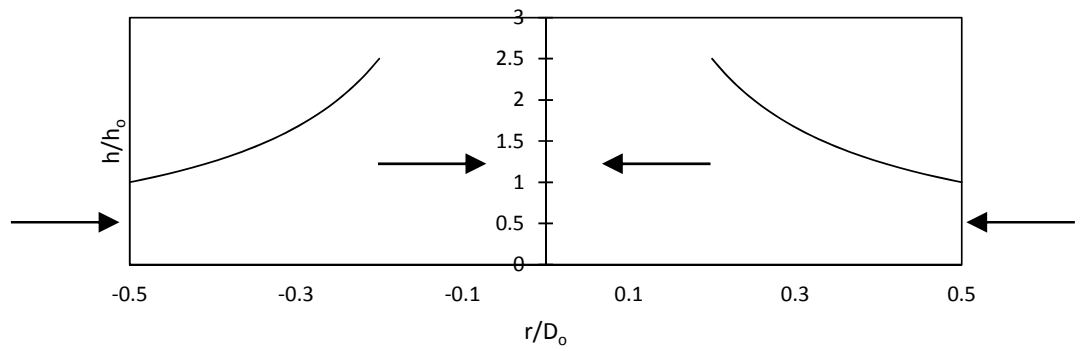

Disk RDE's Aren't Like Annular RDE's!
CFD Video Showing Contours of Temperature

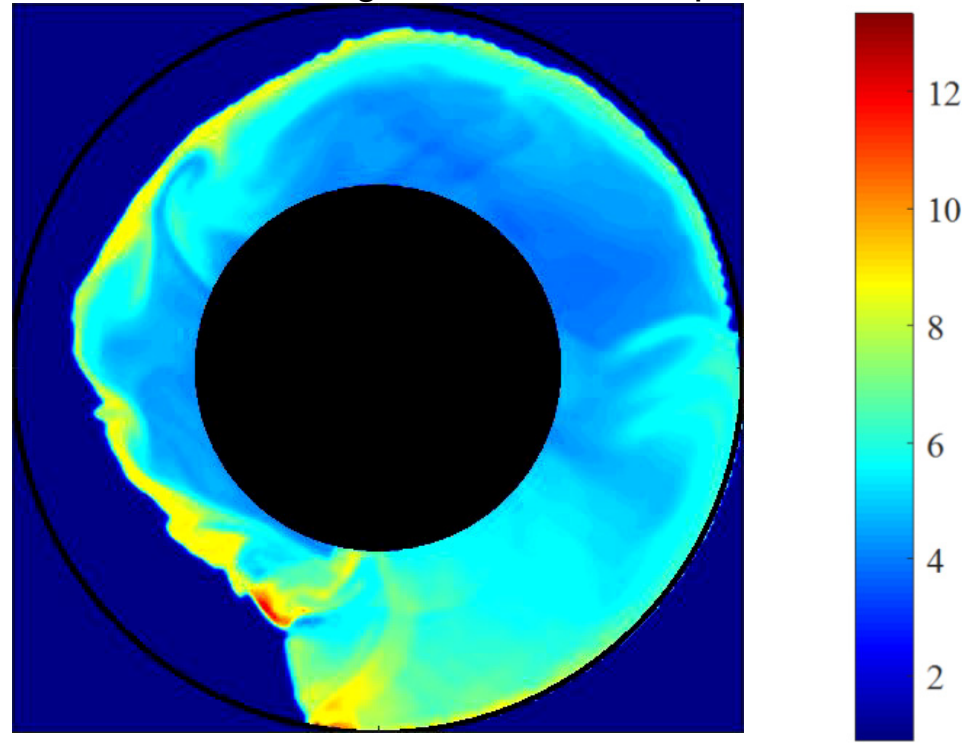

Contours of Temperature 2.25 Revolutions Later

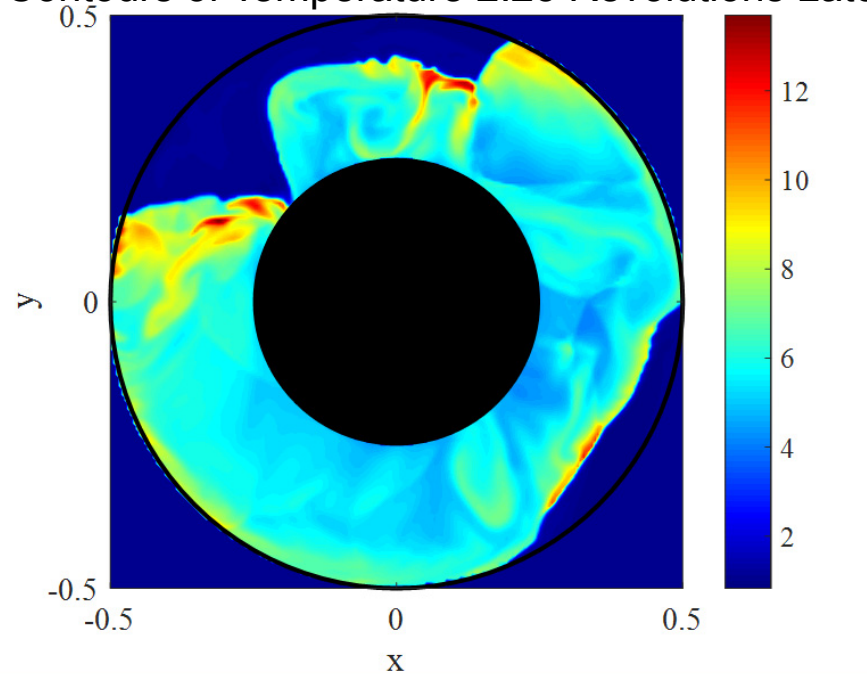




\section{RDE Results: $\mathrm{H}_{2}$ /Air; Radially Inward}

\section{Setup}

- Grid-height variation keeps area constant

- $D_{\mathrm{i}} / \mathrm{D}_{\mathrm{o}}=0.4 ; \mathrm{A}_{\mathrm{in}} / \mathrm{A}_{\mathrm{ch}}=0.6$; Inlet check valve

- Boundary Conditions:

- Outer manifold p, T = 4.0, 1.03846

- Inner diameter $p=1.0$

- Video shows 0.95 time units; started after approximately 10 wave revolutions

Results

- Detonation speed $15 \%$ above CJ based on OD, 54\% below based on ID

- Detonation is stable
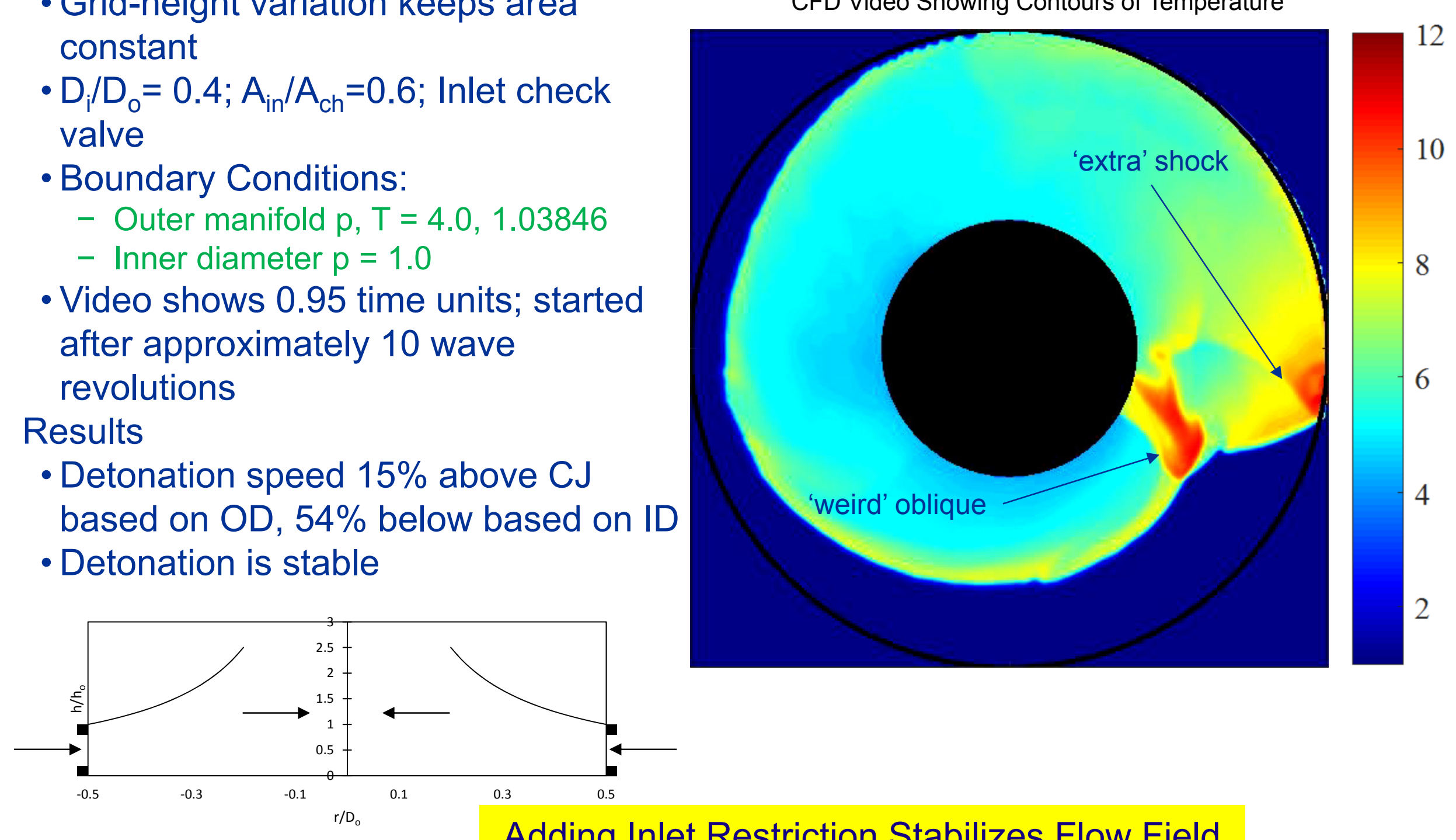

Adding Inlet Restriction Stabilizes Flow Field 
RDE Results: Performance

Observations (Note $-E A P_{i}$ capability not yet implemented)

- Code shows persistent inflow/outflow mismatch of $4 \%$

- Simulation indicates 4\% inflow at outflow (inner) boundary

- Exit flow is highly non-uniform

Temperature Contours With Boundary Velocity Superimposed

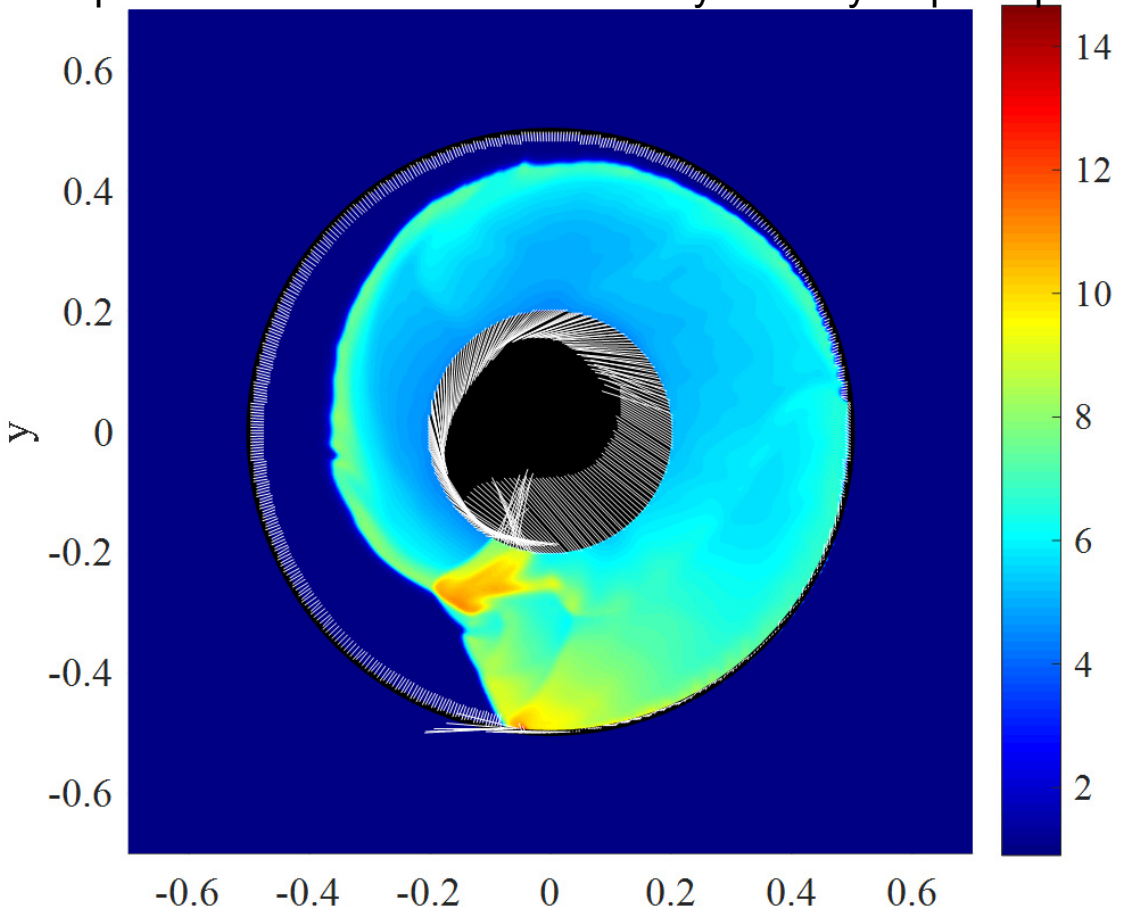

Annular RDE

$\mathrm{T}_{\text {tout }}=7.22$ (theory $=7.22$ )

$\mathrm{EAP}_{\text {ent }}=5.90$ (entropy flux avg.)

PRESSURE GAIN ent $=48 \%$

PRESSURE GAIN $_{\text {EAPi }}=17 \%$

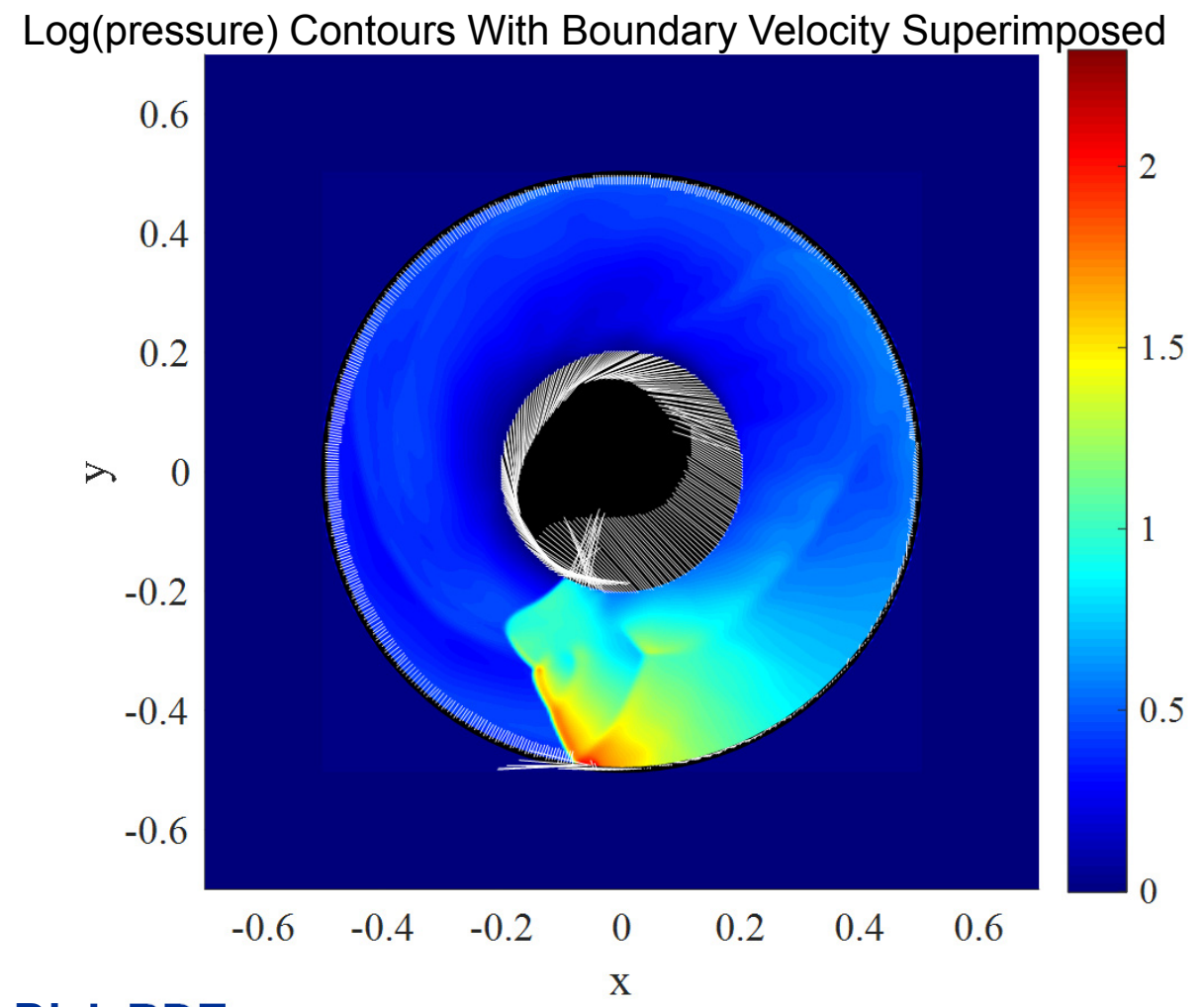

Disk RDE

$\mathrm{T}_{\text {tout }}=7.22$ (theory=7.22)

$\mathrm{EAP}_{\text {ent }}=9.01$ (entropy flux avg.)

PRESSURE GAIN ent $=125 \%$ !!

Radially Inward Disk Vastly

Outperforms Annular RDE IMPLIED PRESSURE GAIN ${ }_{\text {EAPi }}=78 \%$ !! 
Setup

\section{RDE Results: $\mathrm{H}_{2}$ /Air; Radially Outward}

- Grid-height variation keeps area constant

- $D_{\mathrm{i}} / \mathrm{D}_{\mathrm{o}}=0.4 ; \mathrm{A}_{\mathrm{in}} / \mathrm{A}_{\mathrm{ch}}=0.3$; Inlet check valve

-Boundary Conditions:

- Inner manifold p, T = 4.0, 1.03846

- Outer diameter $p=1.0$

- Video shows 0.74 time units; started after approximately 5 wave revolutions Results

- Detonation speed $55 \%$ above CJ based on OD, $38 \%$ below based on ID

- Detonation is stable

- $A_{\text {in }} / A_{c h}=0.6$ results in spilled fuel

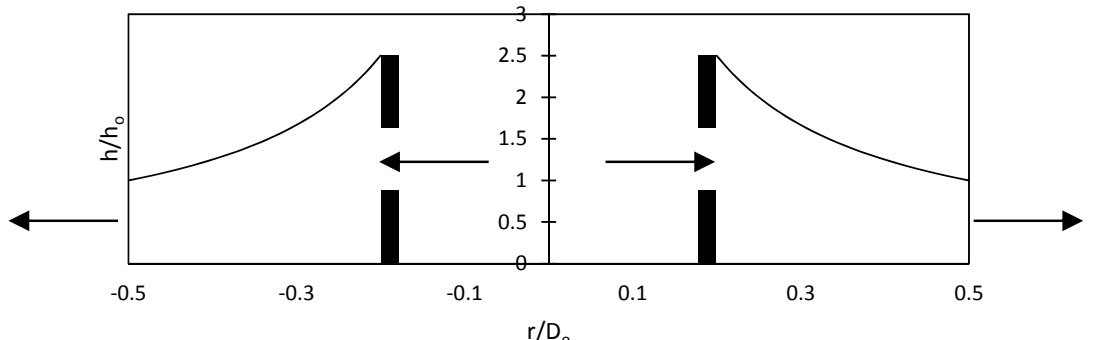

Substantial Inlet Restriction Prevents Fuel Spillage Caused by High Throughflow 


\section{RDE Results: Performance}

\section{Observations (Note $-E A P_{i}$ capability not yet implemented)}

- Code shows persistent inflow/outflow mismatch of $4 \%$

- Simulation indicates 1\% inflow at outflow (outer) boundary

- Exit flow is highly non-uniform

Temperature Contours With Boundary Velocity Superimposed

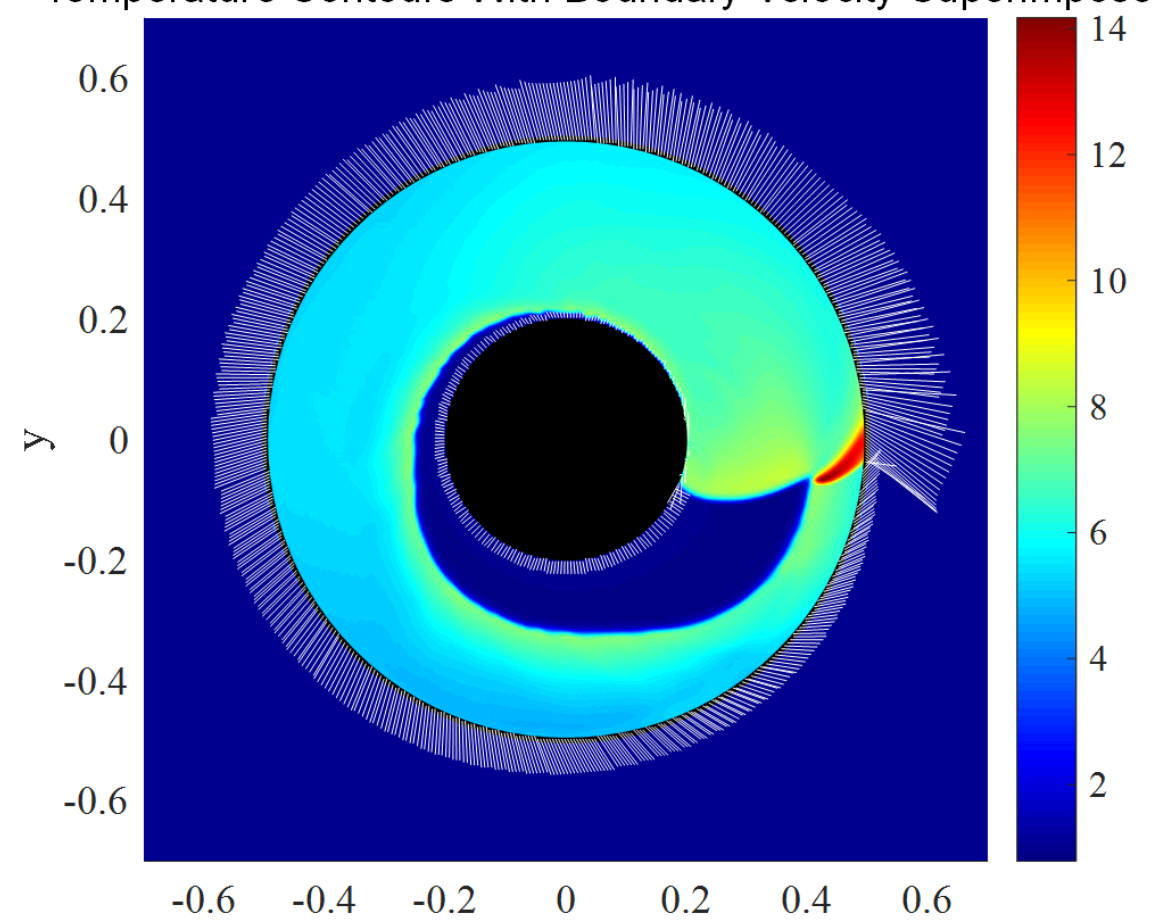

Annular RDE

$\mathrm{T}_{\text {tout }}=7.22$ (theory $=7.22$ )

$\mathrm{EAP}_{\text {ent }}=3.33$ (entropy flux avg.)

PRESSURE GAIN ent $=-16 \%$

PRESSURE GAIN $_{\text {EAPi }}=-32 \%$

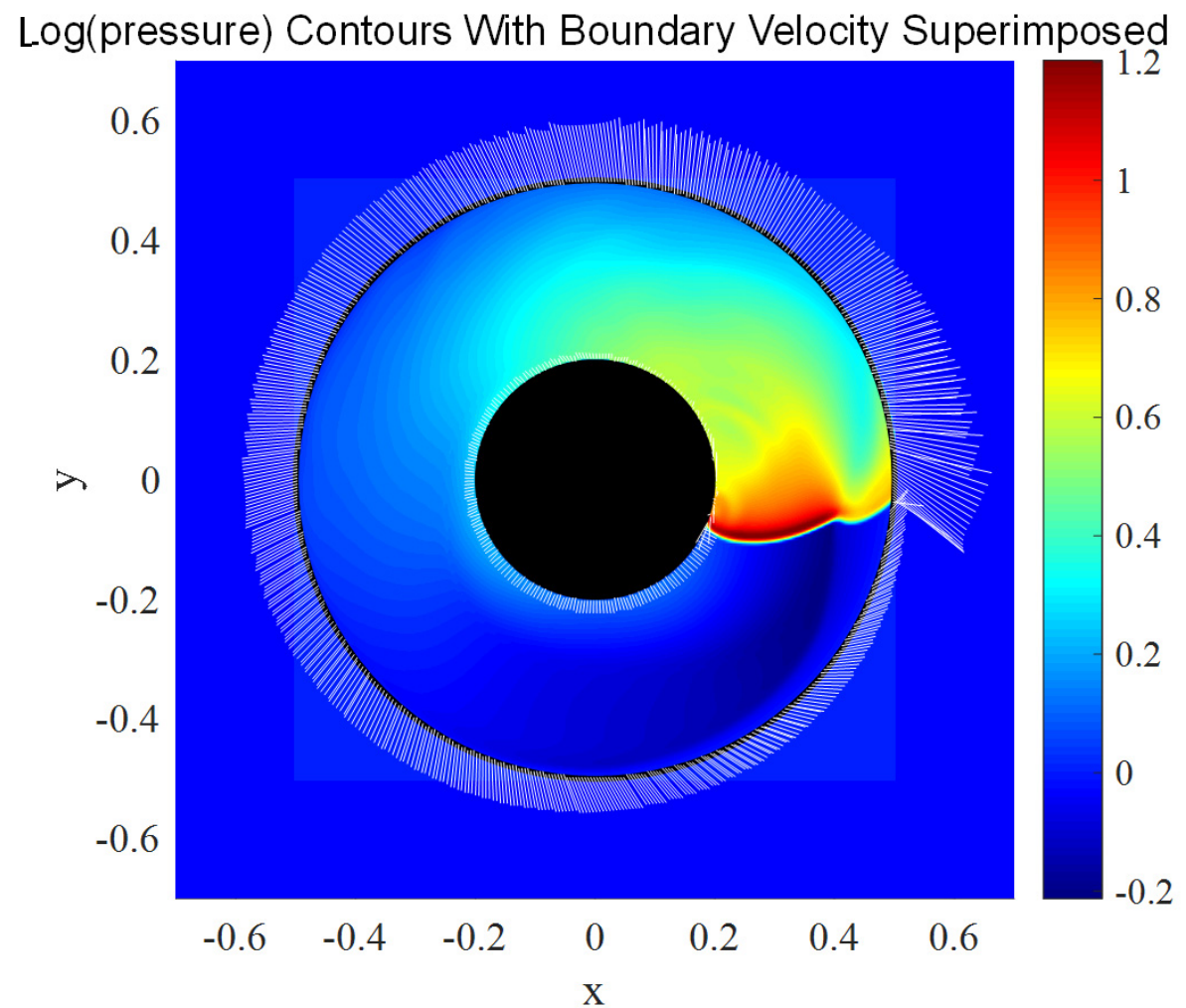

Disk RDE

$\mathrm{T}_{\text {tout }}=7.12$ (theory $=7.22$ )

$\mathrm{EAP}_{\text {ent }}=3.68$ (entropy avg.) PRESSURE GAIN ${ }_{\text {ent }}=-8 \%$ !! IMPLIED PRESSURE GAIN ${ }_{\text {EAPi }}=-26 \%$ !! 


\section{Concluding Remarks}

- Disk RDE configuration successfully simulated using modified NASA simplified Q2D code

- Results are not yet validated, but seem to make sense

- Flow field is quite different from annular configurations

- Based on idealized inlet (i.e. no backflow), adiabatic, inviscid flow

- Radially inward configurations perform substantially better than conventional annular configurations

- Radially inward configurations perform substantially better than radially outward configurations

- Next steps

- Solve boundary mass flow rate mismatch problem (not fundamental)

- Refine wall boundary conditions

- Add EAP ${ }_{i}$ capability

- Add inlet backflow model

- Add heat transfer and friction models

- Validate using AFRL Data

- Perform parametric optimization

- One configuration change has already yielded a 10\% improvement over what has been presented here

- Currently planned for presentation at SciTech 2020 